\title{
Modeling of a photoplethysmographic (PPG) waveform through monte carlo as a method of deriving blood pressure in individuals with obesity.
}

Boonya-Ananta, Tananant; Rodriguez, Andres J.; Hansen, Anders K.; Hutcheson, Joshua D.; RamellaRoman, Jessica C.

\section{Published in:}

Proceedings of SPIE

Link to article, DOI:

$10.1117 / 12.2546589$

Publication date:

2020

Document Version

Publisher's PDF, also known as Version of record

Link back to DTU Orbit

Citation (APA):

Boonya-Ananta, T., Rodriguez, A. J., Hansen, A. K., Hutcheson, J. D., \& Ramella-Roman, J. C. (2020). Modeling of a photoplethysmographic (PPG) waveform through monte carlo as a method of deriving blood pressure in individuals with obesity. In B. L. Ibey, \& N. Linz (Eds.), Proceedings of SPIE (Vol. 11238). [112380A] SPIE International Society for Optical Engineering. Progress in Biomedical Optics and Imaging - Proceedings of SPIE https://doi.org/10.1117/12.2546589

\section{General rights}

Copyright and moral rights for the publications made accessible in the public portal are retained by the authors and/or other copyright owners and it is a condition of accessing publications that users recognise and abide by the legal requirements associated with these rights.

- Users may download and print one copy of any publication from the public portal for the purpose of private study or research.

- You may not further distribute the material or use it for any profit-making activity or commercial gain

- You may freely distribute the URL identifying the publication in the public portal 


\section{Modeling of a photoplethysmographic (PPG) waveform through monte carlo as a method of deriving blood pressure in individuals with obesity.}

Boonya-ananta, Tananant, Rodriguez, Andres, Hansen, Anders, Hutcheson, Joshua, Ramella-Roman, Jessica

Tananant Boonya-ananta, Andres J. Rodriguez, Anders K. Hansen, Joshua D. Hutcheson, Jessica C. Ramella-Roman, "Modeling of a photoplethysmographic (PPG) waveform through monte carlo as a method of deriving blood pressure in individuals with obesity.," Proc. SPIE 11238, Optical Interactions with Tissue and Cells XXXI, 112380A (20 February 2020); doi: 10.1117/12.2546589

SPIE. Event: SPIE BiOS, 2020, San Francisco, California, United States 


\title{
Modeling of a Photoplethysmographic (PPG) Waveform through Monte Carlo as a method of deriving Blood Pressure in Individuals with Obesity
}

\author{
Tananant Boonya-ananta*a, Andres J. Rodriguez ${ }^{\mathrm{a}}$, Anders K. Hansen ${ }^{\mathrm{b}}$, Joshua D. Hutcheson ${ }^{\mathrm{a}}$, \\ Jessica C. Ramella-Roman ${ }^{\mathrm{a}, \mathrm{c}}$ \\ ${ }^{a}$ Department of Biomedical Engineering, Florida International University, $10555 \mathrm{~W}$ Flagler St, \\ Miami, FL, USA 33174; ' Department of Photonics Engineering, Technical University of Denmark, \\ Frederiksborgvej 399, 4000 Roskilde, Denmark; ' ${ }^{\circ}$ Herbert Wertheim College of Medicine, Florida \\ International University, 11200 SW $8^{\text {th }}$ St, Miami, FL, USA 33199
}

\begin{abstract}
Systolic and diastolic blood pressure values can be used as an indicator of an individual's risk for cardiovascular disease. The common practice of blood pressure (BP) measurement using a cuff-based system provides a snapshot of blood pressure at a single instance in time and can be inconvenient and intrusive. The development of optical methods to determine blood pressure could provide continuous monitoring of blood pressure through techniques such as pulse transit time (PTT) or pulse arrival time (PAT) when used with echocardiogram. Cuff based BP devices are known to have variation and inaccuracies when applied to larger arm sizes as seen in individuals with obesity but little is known of the influence of obesity in the PPG/PTT and PAT signals. We propose that accurate waveform replication is required for the derivation of blood pressure applied to individuals with obesity. Here we use the Monte Carlo framework to develop the PPG waveform as a means to derive blood pressure through cuff less techniques. The development of a simulated waveform incorporates realistic changes in the artery related to its biomechanical properties as a pressure wave is propagated through the vessel. It is shown that a change in vessel pressure and geometry directly affects the captured optical signal. The system can account for variations in body-mass index to compensate for geometrical changes in adipose tissue layer and changes in optical properties.
\end{abstract}

Keywords: Photoplethysmography, Monte Carlo, Photon Transport, Optical Properties, Obesity, Blood Pressure, Biomechanics, Cardiovascular Disease,

\section{BACKGROUND}

Obesity is one of the fastest growing diseases in the United States. Approximately $40 \%$ of the population in the United States lives with obesity ${ }^{1-3}$. Obesity is defined by the Centers for Disease Control and Prevention (CDC) as having a Body Mass Index (BMI) of over $30 \mathrm{~kg} / \mathrm{m}^{2}$, BMI is the ratio of body mass in kilograms divided by height in meters squared $\left(\mathrm{kg} / \mathrm{m}^{2}\right)^{4-7}$. Obesity increases the risk of development of a variety of chronic conditions and diseases including, but not limited to, cardiovascular disease (CVD), the leading cause of death for men, women, and people of most racial and ethnic groups in the United States ${ }^{8,9}$. One of the key assessments of cardiovascular health is blood pressure. Blood pressure is an indicator for an individual's risk for CVD. The standard technique of blood pressure evaluation using sphygmomanometers and oscillometric devices are effective to a certain extent. These techniques evaluate the blood pressure of an individual at one instant in time and can be intrusive ${ }^{10}$. Cuff-based blood pressure measurement are known to have variations and inaccuracies when applied to larger arm sizes as in in individuals with obesity, as the subcutaneous adipose tissue layer increases in thickness ${ }^{11-13}$. The development of medical devices that account for physiological changes due to obesity is becoming more prevalent.

Due to restrictions of cuff-based devices, measurement techniques using optical means can provide a continuous method of monitoring blood pressure through techniques such as pulse transit time or pulse arrival time when used in conjunction with electrocardiogram (ECG). The research into cuff-less devices and usage of photoplethysmography (PPG) to derive blood pressure is rapidly expanding as commercialized wearable devices are continually emerging and incorporating health monitoring features. However, similarly to standard techniques for blood pressure monitoring, the physiological changes which occur in individuals with obesity must be accounted for in optical devices. Obesity causes changes to the properties of the skin that affect the optical properties and light interaction ${ }^{14}$, but also increasing subcutaneous adipose tissue can

Optical Interactions with Tissue and Cells XXXI, edited by Bennett L. Ibey, Norbert Linz, Proc. of SPIE

Vol. 11238, 112380A · C 2020 SPIE · CCC code: 1605-7422/20/\$21 · doi: 10.1117/12.2546589 
affect the signal obtained with optical devices ${ }^{13}$. The direct relationship between the pulsatile PPG waveform, peripheral representation cardiovascular function, and blood pressure has yet to be fully explored.

Modeling of the PPG waveform is a critical stepping-stone to the understanding and derivation of a wearable blood pressure monitoring technique through optical means for individuals with obesity. A Monte Carlo (MC) framework will be utilized to model the relationship between the physiological behavior and the optical interactions in order to simulate a PPG waveform that is representative of realistic vascular functions.

\subsection{Physiological Behavior}

\section{METHODS}

The cardiac cycle governs the flow of blood from the heart as blood is pumped to the rest of the body. Understanding the translation of these physiological behaviors to an optical platform is critical to developing a robust methodology for monitoring of cardiac function. Translation of the dynamic behavior of vessel wall movement during pressure wave propagation during blood flow requires a more complex model than a hydrostatic pressure vessel representation. Pressure wave propagation through the cardiovascular system can be modeled as fluid flow through an elastic walled tubing ${ }^{15-18}$. The nature of elastic walled tubing creates variation in the flow streamline as a function of position and time. In rigid wall tubing flow, the velocity profile of the fluid flow is maximum at the center and zero at the walls (no-slip boundary condition), the velocity component in the direction of flow is only dependent on the radius and time and the velocity component perpendicular to flow direction is zero. In elastic walled tubing, both the tangential and radial components of the velocities are dependent on the radius, time and position.

This behavior allows for the pulsatile flow behavior seen in the arterial system of the body. Wall motion of the artery is developed by two stresses acting on the wall, tangential shear stress and radial pressure. Pressure changes occurs local then is propagated downstream. This allows for measurable features such as PTT which accounts for the time between pressure wave generation at the heart and when it reaches the periphery.

\subsection{Mechanical Properties}

In the effort to characterize the behavior of the cardiovascular system and its interactions with optical signals, first, the biomechanics of the arterial system must be understood. With the development of wrist based wearable technology in mind, the behavior of peripheral vessels in the wrist and fingers will be of key interest. Large vessel behavior is studied extensively focusing on the aorta, carotid artery, and large coronary arteries ${ }^{19-24}$. Smaller vessels located in the periphery exhibit similar mechanical behaviors to large vessels such as the aorta or carotid artery ${ }^{25,26}$. These properties can be estimated to the periphery using well-documented behaviors of major vessels. Monson et al. differential and tests mechanical behavior of small versus large cerebral vessels both arteries and veins ${ }^{25}$. The behavior of the arteries at varying sizes exhibit similar characteristics.

Khamdaeng et al. demonstrates the mechanical performance of a carotid artery ${ }^{26}$. Deformation of the arterial wall is recorded using ultrasound at various positions to map out specific behaviors during a cardia cycle. The pressure and vessel diameter change demonstrate similar profiles with physical changes lagging the pressure wave propagation. The behavior of arterial wall expansion and contraction is represented by the combined behavior of smooth muscle cells, collagen, and elastin ${ }^{27-29}$. The characteristics seen at the carotid artery ${ }^{26}$ demonstrates similar behavior to smaller cerebral arteries and a more detailed analysis of vessel behavior is vital in developing parameters used for peripheral arteries. Understanding the mechanical behavior of the vessel allows for the modeling of vessel dilation through Finite Element Analysis (FEA) software. The software allows for visualization of the behavior of a simulated arterial wall under applied internal pressure. From the FEA simulation, the expansion of the radial artery was extracted with estimated input mechanical properties $^{12,13,24,26,29}$.

The artery is modeled in Solidworks computer-aided design (CAD) software. Arterial geometric parameters are modeled based on the radial artery located at the volar surface of the forearm where the pulse is commonly palpated. A twodimensional drawing of the vessel with associated dimensions is shown in Figure 1. 


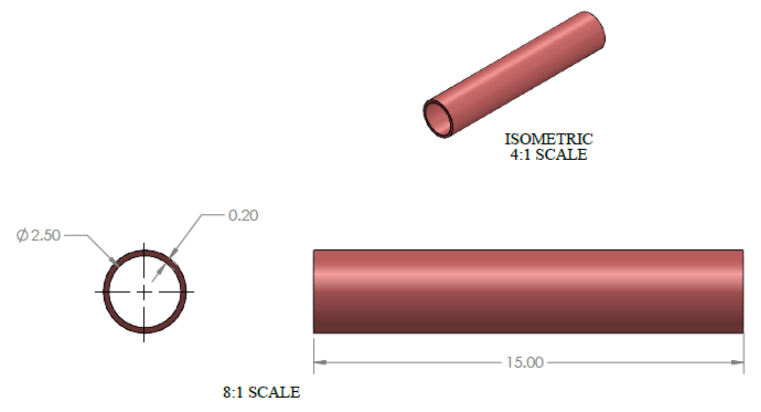

Figure 1. Vessel 2D CAD geometry drawing. This is the model used for the radial artery to understand its behavior during the cardiac cycle. All dimensions in [mm].

Vessel dimensions representing the radial artery are $2.5 \mathrm{~mm}$ inner diameter and 0.2 wall thickness ${ }^{12,13,26}$. From this model of the vessel, mechanical properties are applied. Vessel wall mass density Poisson's ratio are determined to be $1106 \mathrm{~kg} / \mathrm{m}^{3}$ and $0.49^{24,26,29}$ respectively. Arterial mechanical stiffness values range between $0.70 \mathrm{MPa} 1.10 \mathrm{MPa}^{26}$.

\subsection{Monte Carlo Model Adaptation}

The Monte Carlo model is performed using an adapted version of Jacques, S. "mcxyz.c"30 by Marti, D. et al. called MCMatlab $^{31}$. The Monte Carlo model incorporates a $1.4 \mathrm{~cm}$ x $1.4 \mathrm{~cm}$ x $0.8 \mathrm{~cm}$ frame at $200 \times 100 \times 200$ elements in each respective direction. The layer composition of the geometry is shown in Figure 2, associated geometric parameters and optical properties are denoted in Table 1.

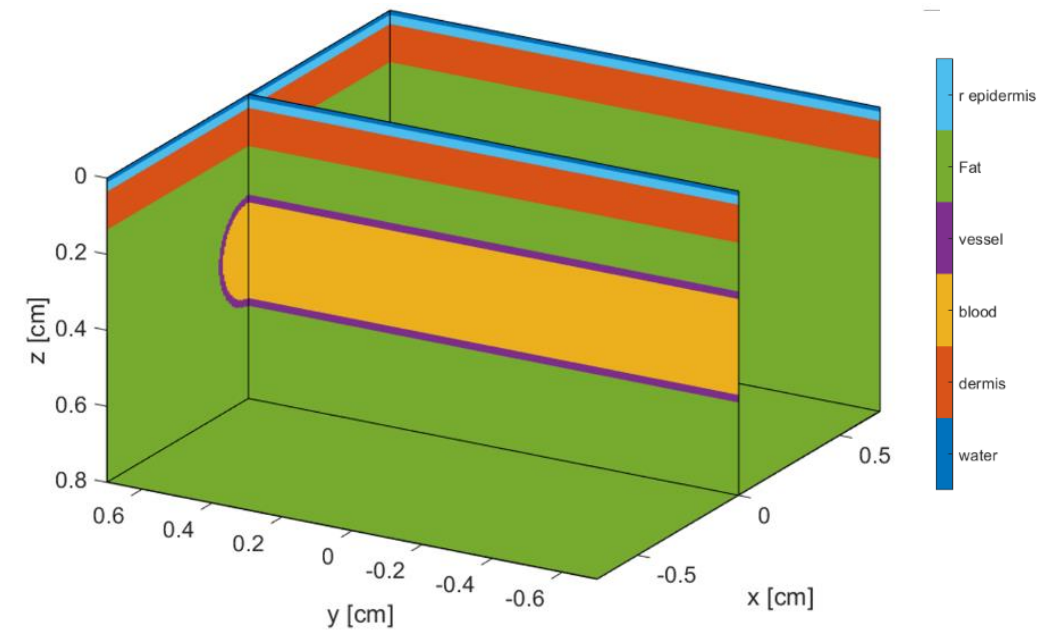

Figure 2. Sample geometric illustration of Monte Carlo input configuration. Layer legend and associated optical properties are shown in Table 1.

Table 1. Optical properties at two wavelengths of layered geometry.

\begin{tabular}{|l|c|c|c|c|}
\hline \multicolumn{1}{|c|}{ Layer } & $\begin{array}{c}\mu_{a}\left[\mathrm{~cm}^{-1}\right] \\
660 \mathrm{~nm} / 890 \mathrm{~nm}\end{array}$ & $\begin{array}{c}\mu_{s}\left[\mathrm{~cm}^{-1}\right] \\
660 \mathrm{~nm} / 890 \mathrm{~nm}\end{array}$ & $\mathrm{~g}$ & $\mathrm{n}$ \\
\hline r Epidermis & $0.3442 / 0.3184$ & $121.2 / 224.7$ & 0.9 & 1.4 \\
Dermis & $0.5453 / 2459$ & $208.6 / 116.7$ & 0.9 & 1.4 \\
Fat & $0.0001 / 0.0217$ & $249.7 / 189.8$ & 0.9 & 1.4 \\
Vessel Wall & $0.8 / 0.8$ & $230 / 230$ & 0.9 & 1.4 \\
Blood & $2.026 / 6.32$ & $75.76 / 56.18$ & 0.9 & 1.4 \\
\hline
\end{tabular}


The epidermal layer is $0.25 \mathrm{~mm}$ thick. This is a modified epidermal layer indicated as "r epidermis". This layer is scaled up to $0.25 \mathrm{~mm}$ from $0.10 \mathrm{~mm}$ in order to increase the number of elements in the mesh for the epidermis. In order to compensate for the increase in size, the optical properties, absorption and scattering coefficients, are reduced by the same factor. The dermis is represented by a single layered dermis at $1.0 \mathrm{~mm}$ thickness. Below the dermis is the subcutaneous adipose tissue with makes up the remainder of the frame. Within this layer, the radial artery is modeled as a cylinder situated at $2.5 \mathrm{~mm}$ depth from the top surface ${ }^{12,13}$. The target radial artery is constructed with a vessel wall and the internal lumen is filled with blood.

Source and detector separation is $1.0 \mathrm{~cm} x$ direction at the surface layer. Simulation photon count was tested at 100 million photons per simulation. Simulations were performed on Windows 10 64-bit Operating System with Intel ${ }^{\circledR}$ Core ${ }^{\text {TM }}$ i7-8700 CPU 3.20GHz, 32GB RAM, NVIDIA GeForce GTX 1070 GPU, and Windows 10 64-bit Operating System with Intel® Core $^{\mathrm{TM}}$ i7-8750H CPU 2.20GHz, 16GB RAM and NVIDIA GeForce GTX 1050 Ti GPU. Simulations speeds range between rates of 2 million photons per minute to 15 million photons per minute.

The results of the finite element simulation for arterial dilation demonstrates a change of $0.20 \mathrm{~mm}$ to $0.50 \mathrm{~mm}$ for developing the primary and secondary features of a PPG waveform. Visual representation of propagated pulse vessel dilation is represented in Figure 3.

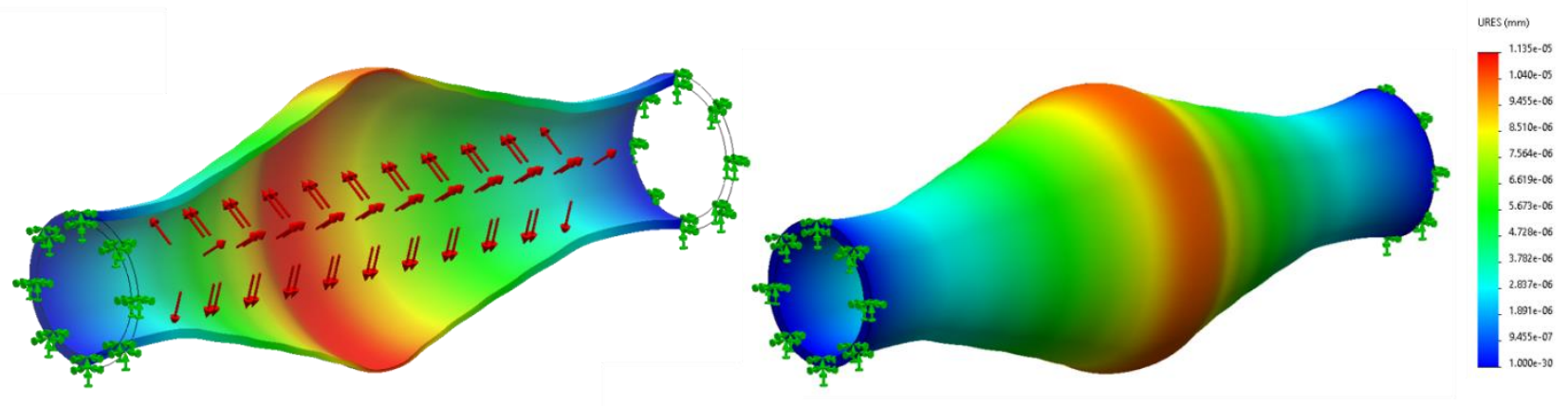

Figure 3. Arterial wall dilation contour plot of total displacement. Red regions show regions of highest displacement and blue for lowest displacement.

The representation shows a single location of pulse propagated vessel shape, this is different from a hydrostatic pressure vessel representation. The change to the vessel geometry significantly affects the change in inner diameter of the vessel yet limited change to vessel wall compression. From this, the vessel is assumed to be relatively incompressible compared to the diametral change under applied pressure.

The adaptation of these FEA results to MC geometry in light transport was done using ellipsoid representation of two pulse shape features often seen in PPG output signal. Two ellipsoid model was represented using Equations 1-3.

$$
\begin{gathered}
\left(\frac{x}{\frac{I D x}{2}}\right)^{2}+\left(\frac{z-d}{\frac{I D x}{2}}\right)^{2}+\left(\frac{y+p L 1}{p w d 1}\right)^{2}=1^{2} \\
\left(\frac{x}{\frac{I D x d}{2}}\right)^{2}+\left(\frac{z-d}{\frac{I D x d}{2}}\right)^{2}+\left(\frac{y+p L 2}{p w d 2}\right)^{2}=1^{2} \\
\text { psep }=p L 1-p L 2
\end{gathered}
$$

Development of these equations is a result of combined understanding of pulsatile flow in elastic walled tubing and arterial biomechanical behavior. In Equations 1 and 2, $x, y$, and $z$ represent each direction in the frame, $d$ locates the ellipsoid in the vertical, $z$, direction in the media. In Equation 1, IDx defines the dilation of the inner diameter of the vessel, $p L 1$ is the location of the primary ellipsoid in the $y$-axis, and $p w d 1$ defines the width of the primary ellipsoid in the $y$ direction. In Equation 2, IDxd represents the dilation of the inner diameter of the secondary ellipsoid, $p L 2$ is the 
location of the secondary ellipsoid in the $y$-axis, and $p w d 2$ defines the width of the secondary ellipsoid in the $y$ direction. Equation 3 defines the $p s e p$ parameter as the separation between the first and second ellipsoids. The geometry developed with these equations is shown in Figure 4.

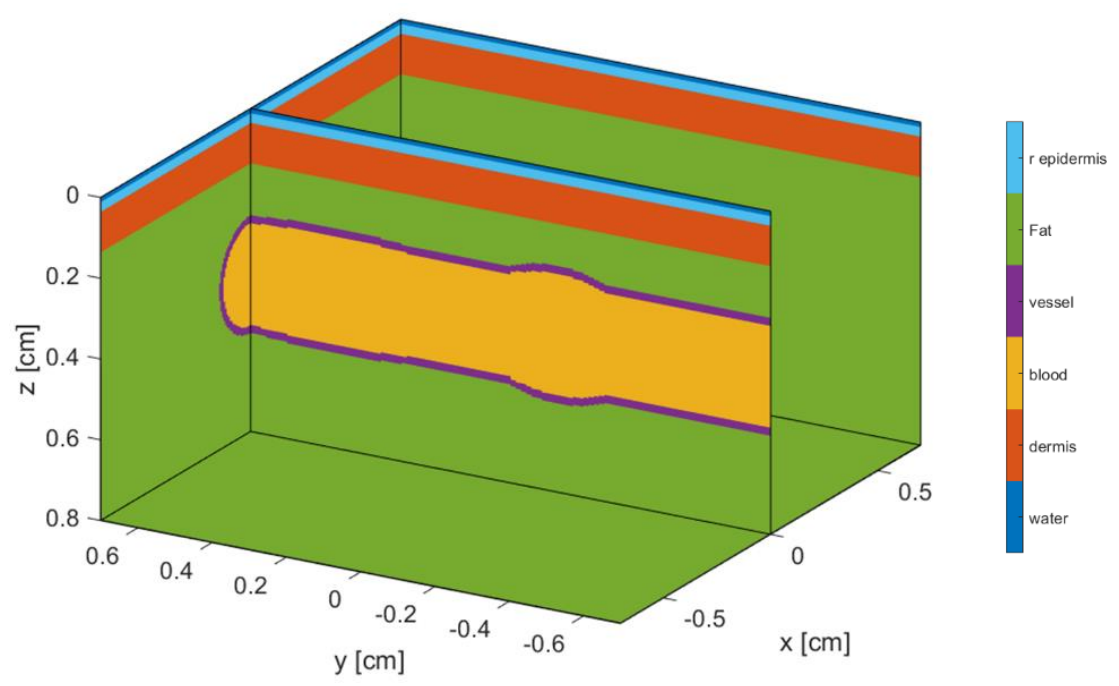

Figure 4. Illustration of pulse shape generating features in Monte Carlo. Geometric parameters of arterial dilation and pressure wave propagation is adapted into Monte Carlo through variation in geometry of the artery. Pulse features are developed through Equations 1-3.

\section{RESULTS}

The Monte Carlo simulation was performed over a set series of positions of the pulse shape generating features in order to capture changes in total reflectance as the pulse travels through the capture frame. Figure 4A and 4B demonstrates a Monte Carlo output of the arterial pulse propagation through the frame of interest.
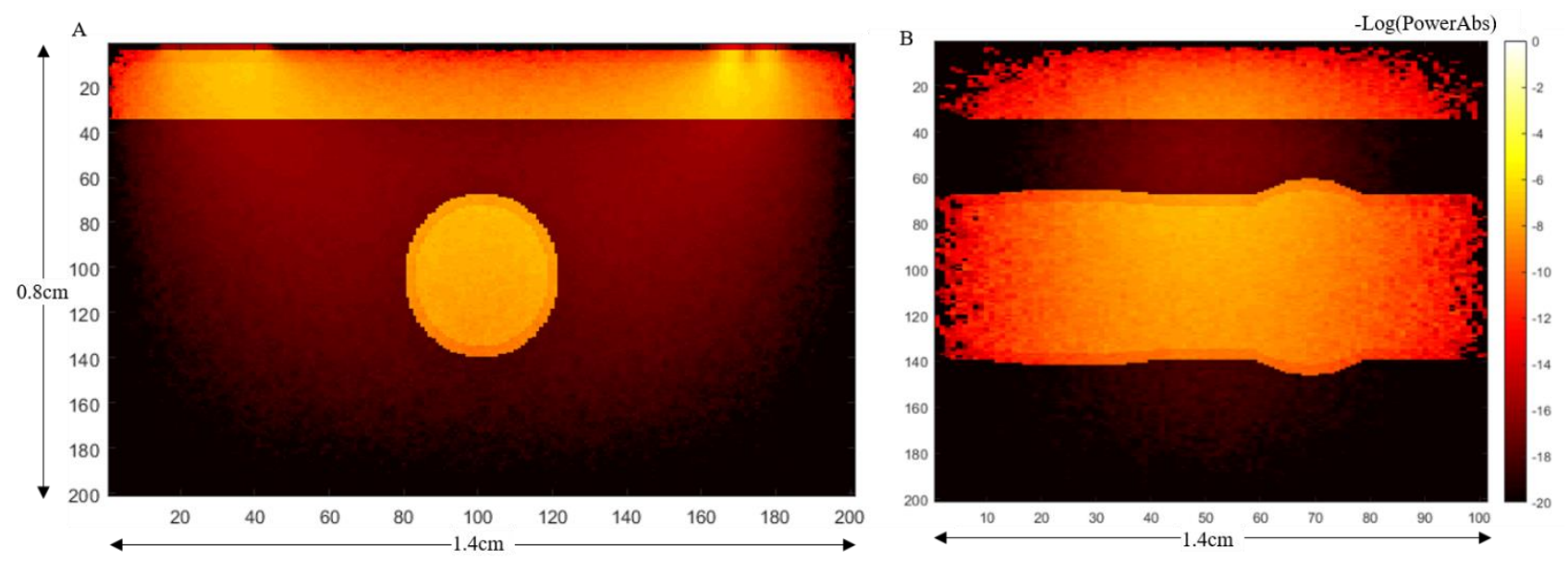

Figure 5. Artery front (A) and side (B) view Monte Carlo image output. Plot represents the negative logarithm of power absorbed at each unit volume.

The Monte Carlo geometry captures a $1.4 \mathrm{~cm} \times 1.4 \mathrm{~cm}$ x $0.8 \mathrm{~cm}$ area isolated for the radial artery. Figure 5 shows a single position front and side view of the artery as light is injected into medium and pulse shape generating geometry is moved through the frame. The relative change in captured reflectance signal at the detector is used to generate the pulse waveform. The waveform is shown in Figure 6. 


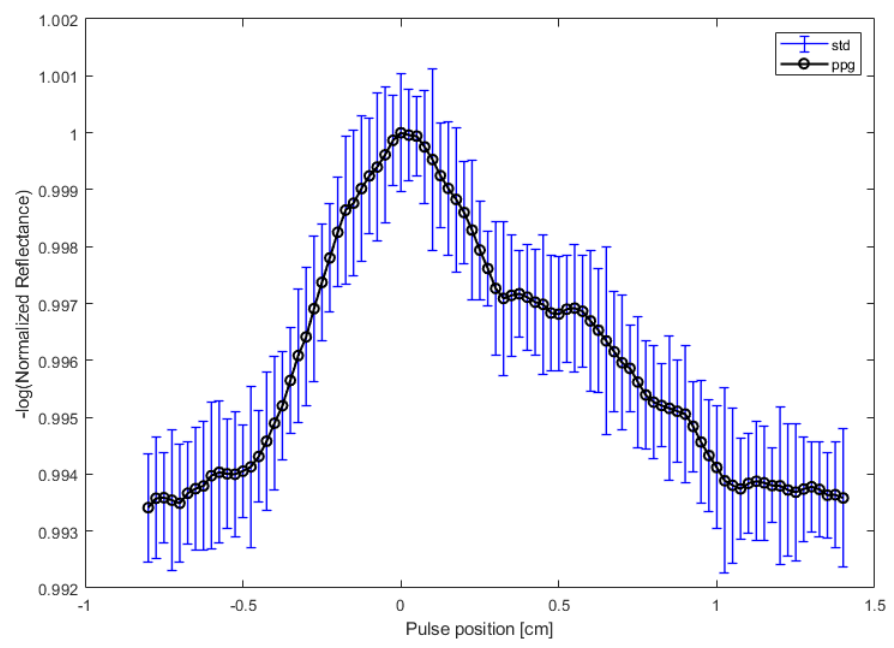

Figure 6. Normalized Reflectance Signal. Simulated PPG waveform at $660 \mathrm{~nm}$ wavelength and $2.50 \mathrm{~mm}$ vessel depth.

Simulated PPG signal shown in Figure 6 is created using 89 positions of the ellipsoid moving from negative $y$ position to positive $y$ positions at 100 million photons per position. The single waveform generation was performed 15 times in order to develop a set of mean and standard deviation for each value. The plot represents a normalized negative log of the reflectance signal captured at the detector. The average standard deviation is 0.0011 and maximum standard deviation is 0.002 . This preliminary test of generating a simulated waveform shows promising results being able to show both primary and secondary peak features.

Adaptation of model to individuals with obesity shows changes to location of the radial artery as subcutaneous adipose tissue increases. The artery is moved down from $2.5 \mathrm{~mm}$ to $3.5 \mathrm{~mm}$ in depth. The changes to the capture reflectance signal is shown in Figure 7.

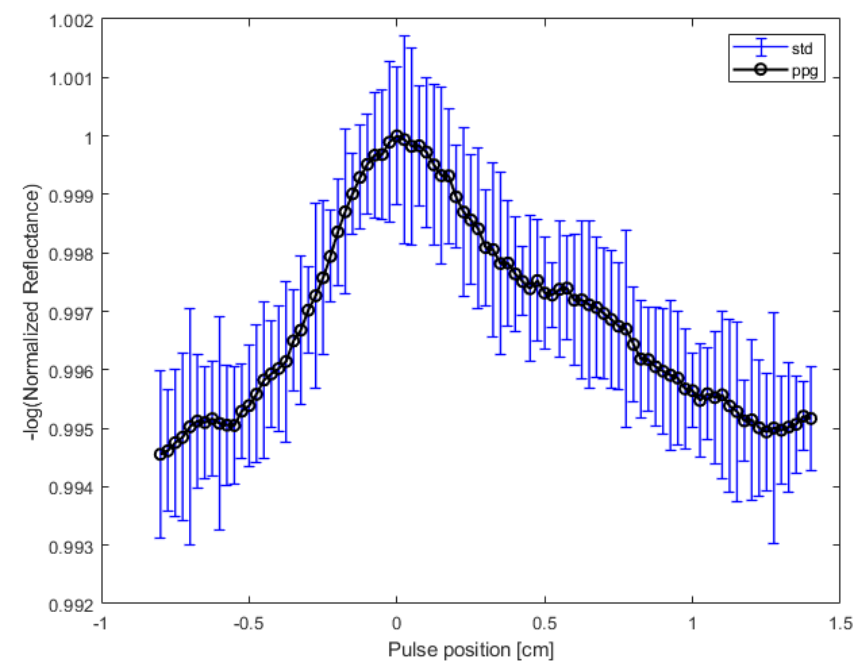

Figure 7. Normalized Reflectance Signal. Simulated PPG waveform at $660 \mathrm{~nm}$ wavelength and $3.50 \mathrm{~mm}$ vessel depth simulating signal from individual with obesity. 
Figure 7 shows the simulated PPG signal with increased subcutaneous layer thickness and a deeper radial artery location. Vessel depth is $3.5 \mathrm{~mm}$. The waveform was generated using 89 positions of the ellipsoids with 100 million photons per position over 15 iterations. The average standard deviation is 0.0012 and the maximum standard deviation is 0.0021 . In this case, both primary and secondary peak features are seen in the simulated PPG signal. However, the total range from baseline to maximum sees a smaller relative change and the secondary feature appears less prominent.

\section{CONCLUSION}

The effort to develop a system for wearable optical methods of estimating blood pressure for individuals with obesity, understanding the connection of vascular biomechanics and its interaction with optical technology is a critical steppingstone. A method of monitoring vascular health for both the patient and the physicians is essential to work the intervention of cardiovascular disease. Simulation of the photoplethysmographic waveform using a Monte Carlo framework can provide the link between the two domains. From previous work performed to quantify arterial biomechanics, geometric features of radial arterial expansion and contraction under pulsatile flow induced during the cardiac cycle are converted into parameters for a Monte Carlo light transport model. Reflectance signal collected at a detector is then converted into a simulated PPG waveform generated for a single cardia cycle.

Initial simulations show differences between wave as well as arterial depth representing a thicker subcutaneous fat layer as degrees of obesity increases. Correlations to specific cardiovascular properties can be used to generate simulated waveform modeling different vascular health states. Waveform peak is correlated to systolic behavior; yet secondary waveform peak is still not clearly quantified.

\section{ACKNOWLEDGEMENTS}

This work was supported by the National Science Foundation Engineering Research Center for Precise Advanced Technologies and Health Systems for Underserved Populations (PATHS-UP) (\#1648451). 


\section{REFERENCES}

1 Hales, C. M., Carroll, M. D., Fryar, C. D. \& Ogden, C. L. Prevalence of obesity among adults and youth: United States, 2015-2016. (2017).

2 Finkelstein, E. A., Trogdon, J. G., Cohen, J. W. \& Dietz, W. Annual Medical Spending Attributable To Obesity: Payer-And Service-Specific Estimates: Amid calls for health reform, real cost savings are more likely to be achieved through reducing obesity and related risk factors. Health affairs 28, w822w831 (2009).

3 Ogden, C. L. et al. Prevalence of obesity among adults, by household income and education-United States, 2011-2014. MMWR. Morbidity and mortality weekly report 66, 1369 (2017).

4 Garrow, J. S. \& Webster, J. Quetelet's index (W/H2) as a measure of fatness. International journal of obesity 9, 147-153 (1985).

5 Freedman, D. S., Horlick, M. \& Berenson, G. S. A comparison of the Slaughter skinfold-thickness equations and BMI in predicting body fatness and cardiovascular disease risk factor levels in children. The American journal of clinical nutrition 98, 1417-1424 (2013).

6 Flegal, K. M. \& Graubard, B. I. Estimates of excess deaths associated with body mass index and other anthropometric variables. The American journal of clinical nutrition 89, 1213-1219 (2009).

7 Willett, K., Jiang, R., Lenart, E., Spiegelman, D. \& Willett, W. Comparison of bioelectrical impedance and BMI in predicting obesity-related medical conditions. Obesity 14, 480-490 (2006).

8 Heron, M. P. Deaths: leading causes for 2017. (2019).

9 Santulli, G. Epidemiology of cardiovascular disease in the 21st century: updated numbers and updated facts. J Cardiovasc Dis 1, 1-2 (2013).

10 James, G. D. Blood pressure response to the daily stressors of urban environments: Methodology, basic concepts, and significance. American Journal of Physical Anthropology 34, 189-210 (1991).

11 Van Beekvelt, M., Borghuis, M., Van Engelen, B., Wevers, R. \& Colier, W. Adipose tissue thickness affects in vivo quantitative near-IR spectroscopy in human skeletal muscle. Clinical science 101, 2128 (2001).

12 Kim, J. U., Lee, Y. J., Lee, J. \& Kim, J. Y. Differences in the properties of the radial artery between Cun, Guan, Chi, and nearby segments using ultrasonographic imaging: a pilot study on arterial depth, diameter, and blood flow. Evidence-Based Complementary and Alternative Medicine 2015 (2015).

13 Lee, B. J., Jeon, Y. J. \& Kim, J. Y. Association of obesity with anatomical and physical indices related to the radial artery in Korean adults. European Journal of Integrative Medicine 14, 22-27 (2017).

14 Yosipovitch, G., DeVore, A. \& Dawn, A. Obesity and the skin: skin physiology and skin manifestations of obesity. Journal of the American Academy of Dermatology 56, 901-916 (2007).

15 Zamir, M. \& Budwig, R. Physics of pulsatile flow. Appl. Mech. Rev. 55, B35-B35 (2002).

16 Shigley, J. E. Shigley's mechanical engineering design. (Tata McGraw-Hill Education, 2011).

17 Zamir, M. Hemo-dynamics. (Springer, 2016).

18 Attinger, E. The physics of pulsatile blood flow with particular reference to small vessels. Investigative Ophthalmology \& Visual Science 4, 973-987 (1965).

19 Lam Po Tang, E. J. et al. Non-contact Quantification of Jugular Venous Pulse Waveforms from Skin Displacements. Sci Rep 8, 17236, doi:10.1038/s41598-018-35483-4 (2018).

20 Moxham, I. M. Understanding Arterial Pressure Waveforms. Southern African Journal of Anaesthesia and Analgesia 9, 40-42, doi:10.1080/22201173.2003.10872991 (2014).

21 Denardo, S. J., Nandyala, R., Freeman, G. L., Pierce, G. L. \& Nichols, W. W. Pulse wave analysis of the aortic pressure waveform in severe left ventricular systolic dysfunction. Circ Heart Fail 3, 149156, doi:10.1161/CIRCHEARTFAILURE.109.862383 (2010).

22 Xiao, H., Tan, I., Butlin, M., Li, D. \& Avolio, A. P. Arterial viscoelasticity: role in the dependency of pulse wave velocity on heart rate in conduit arteries. Am J Physiol Heart Circ Physiol 312, H1185H1194, doi:10.1152/ajpheart.00849.2016 (2017). 
23 Truijers, M. et al. Wall stress analysis in small asymptomatic, symptomatic and ruptured abdominal aortic aneurysms. Eur J Vasc Endovasc Surg 33, 401-407, doi:10.1016/j.ejvs.2006.10.009 (2007).

24 Peterson, L. H., Jensen, R. E. \& Parnell, J. Mechanical properties of arteries in vivo. Circulation Research 8, 622-639 (1960).

25 Monson, K. L., Goldsmith, W., Barbaro, N. M. \& Manley, G. T. Significance of source and size in the mechanical response of human cerebral blood vessels. Journal of biomechanics 38, 737-744 (2005).

26 Khamdaeng, T., Luo, J., Vappou, J., Terdtoon, P. \& Konofagou, E. E. Arterial stiffness identification of the human carotid artery using the stress-strain relationship in vivo. Ultrasonics 52, 402-411, doi:10.1016/j.ultras.2011.09.006 (2012).

27 Humphrey, J. \& Rajagopal, K. A constrained mixture model for growth and remodeling of soft tissues. Mathematical models and methods in applied sciences 12, 407-430 (2002).

28 Holzapfel, G. A. \& Ogden, R. W. Constitutive modelling of arteries. Proceedings of the Royal Society A: Mathematical, Physical and Engineering Sciences 466, 1551-1597, doi:10.1098/rspa.2010.0058 (2010).

29 Van de Vosse, F. \& Van Dongen, M. Cardiovascular fluid mechanics-lecture notes. Faculty of Applied Physics, Faculty of Mechanical Engineering, Eindhoven University of Technology, Eindhoven, Netherlands (1998).

30 Jacques, S. \& Li, T. Monte Carlo simulations of light transport in 3D heterogenous tissues (mcxyz. c). See http://omlc. org/software/mc/mcxyz/index. html [accessed 30.01. 2017] (2013).

31 Marti, D., Aasbjerg, R. N., Andersen, P. E. \& Hansen, A. K. MCmatlab: an open-source, user-friendly, MATLAB-integrated three-dimensional Monte Carlo light transport solver with heat diffusion and tissue damage. Journal of biomedical optics 23, 121622 (2018). 